\title{
SIZE EFFECTS IN METALLIC FILMS
}

\author{
F. WARKUSZ \\ Wroclaw Technical University, Institute of Physics, \\ 50-370 Wroclaw, Wybrzeże Wyspiańskiego 27, Poland
}

(Received June 15, 1977)

\begin{abstract}
Based on the theories so far developed, an approximate expression for the resistivity of metallic films is derived, which takes into account film thickness, grain diameter, as well as the coefficients of surface scattering and grain boundary scattering. The derived formulae are compared with the results achieved by other authors.
\end{abstract}

\section{INTRODUCTION}

The investigations carried out within the last few years allow for the conclusion that the resistivity of metallic films is influenced not only by isotropic electron scattering in the volume of the film and surface scattering, but also by grain-boundary scattering effects. The Fuchs-Sondheimer theory ${ }^{1,2}$ takes into account isotropic scattering and surface scattering, whereas Mayadas and Shatzkes ${ }^{3,4}$ have considered the simultaneous action of isotropic electron scattering, surface scattering and grainboundary scattering. Wissmann and Wedler ${ }^{5,6}$ have derived an analytical expression for the electrical resistivity of thin polycrystalline metal films. This expression also includes the grain-boundary scattering effect. The correlation between the Mayadas-Shatzkes and the Wissmann-Wedler models was studied by F. Thieme and W. Kirstein. ${ }^{7}$ E. E. Mola and J. M. Heras, ${ }^{8-1} 0$ using the wellknown Mayadas-Shatzkes model, have derived exact and approximate equations for the thickness dependence of resistivity. These equations take into account thick films with grain diameter equal to the film thickness. Based on the Mola-Heras approach, ${ }^{9}$ C. R. Tellier and A. J. Tosser ${ }^{11,12}$ have established the approximate expression for the electrical resistivity of very thin polycrystalline metallic films. Recently, L. A. Moraga and A. Vilche ${ }^{13}$ have supplemented the Fuchs-Sondheimer theory, ${ }^{1,2}$ by consideration of the layer model of an inhomogeneous film. In exceptional cases, the expression is reduced to the Fuchs relation.

\section{APPROXIMATE EXPRESSIONS FOR THE RESISTIVITY OF METAL FILMS}

The well-known electrical conductivity model of Fuchs $^{1}$ and Sondheimer ${ }^{2}$ describes the bulk resistivity $\left(\rho_{\mathrm{B}}\right)$ to film resistivity $\left(\rho_{\mathrm{F}}\right)$ relationship in terms of the following equation:

$$
\frac{\rho_{F}}{\rho_{B}}=\frac{1}{F(k, p)}
$$

where

$$
\begin{aligned}
F(k, p)= & 1-\frac{3}{2 k}(1-p) \\
& \int_{1}^{\infty}\left(\frac{1}{t^{\prime 3}}-\frac{1}{t^{\prime 5}}\right) \frac{1-\exp \left(-k t^{\prime}\right)}{1-p \exp \left(-k t^{\prime}\right)} d t^{\prime}
\end{aligned}
$$

is the Fuchs' model $;^{1} k=t / \lambda, t$ refers to the film thickness, $1 / t^{\prime}=\cos \theta, \lambda$ is the background mean free path, and $p$ denotes the reflection coefficient at the film surface. In Eq. (2) both the thickness $t$ and the reflection coefficient $p$ are accounted for.

Mayadas, Shatzkes and Janak, ${ }^{3}$ considering the grain-boundary dependence of the film resistivity, derive the following relations

$$
\frac{\rho_{B}}{\rho_{o}}=\frac{1}{G(\alpha)}
$$

where $\rho_{o}$ is the resistivity of the single crystal, 
100

$$
G(\alpha)=1-\frac{3}{2} \alpha+3 \alpha^{2}-3 \alpha^{3} \ln \left(1+\frac{1}{\alpha}\right)
$$

and

$$
\alpha=\frac{1}{s} \frac{r}{1-r}
$$

where $s=d / \lambda, d$ is the grain diameter, and $r$ the grainboundary reflection coefficient. ${ }^{3}$

Calculating $\rho_{B}$ from Eq. (3) and substituting into Eq. (1) we obtain

$$
\frac{\rho_{F}}{\rho_{0}}=\frac{1}{F(k, p) G(\alpha(r, s))}
$$

For high and low values of $k$, Eq. (2) becomes

$$
\frac{1}{F(k, p)}=1+\frac{3}{8 k}(1-p) \text { when } k>1
$$

and

$$
\frac{1}{F(k, p)}=\frac{4}{3} \frac{1-p}{1+p} \frac{1}{k \ln 1 / k} \text { when } k<1
$$

For very low and very high values of $\alpha$, the $G(\alpha)$ function $^{4}$ is as follows:

$$
\frac{1}{G(\alpha)}=1+\frac{3}{2} \alpha \text { when } \alpha \ll 1
$$

and

$$
\frac{1}{G(\alpha)}=\frac{4}{3} \alpha \text { when } \alpha \gg 1
$$

Let us consider four forms of Eq. (6), with the functions $F(k, p)$ and $G(\alpha)$ expressed in terms of Eq. (7), (8), (9), and (10).

\subsection{Thick Film with Large Grains $(k>1, \alpha \ll 1)$}

Substituting Eq. (7) and (9) into Eq. (6) we obtain

$$
\begin{aligned}
& \rho_{F}=\rho_{0}+\rho_{d}(\alpha \ll 1)+\rho_{t}(k>1)+ \\
& \rho_{c}(k>1, \alpha \ll 1)
\end{aligned}
$$

is the resistivity depending on the grain-boundary scattering (internal size effect);

$$
\rho_{t}=\frac{3}{8} \frac{1-p}{k} \rho_{0}
$$

denotes the resistivity depending on the surface scattering (external size effect);

$$
\rho_{c}=\frac{9(1-p) r}{16 k s(1-r)} \rho_{0} \text { or } \rho_{c}=\frac{\rho_{d}(\alpha \ll 1) \rho_{t}(k>1)}{\rho_{0}}
$$

Since $\alpha \ll 1$ and $k>1$, it thus follows that $(\alpha / k) \rightarrow 0$. Hence, $\rho_{c}$ is very low and, therefore, negligible.

From Eq. (11), it follows that resistivities depending on different factors sum (according to Matthiessen's rule). When neglecting $\rho_{c}(k>1$, $\alpha \ll 1$ ), the film resistivity becomes the same as that obtained by P. Wissmann ${ }^{6}$ and F. ThiemeW. Kirstein. ${ }^{7}$

\subsection{Thin Monocrystalline Film $(k<1, \alpha \ll 1, r \rightarrow 0)$}

By substituting Eq. (8) and (9) into Eq. (6) we get

$$
\rho_{F}=\rho_{t}(k<1)+\rho_{c}(k<1, \alpha \ll 1)
$$

where

$$
\rho_{t}=\frac{4}{3} \frac{1-p}{1+p} \frac{1}{k \ln 1 / k} \rho_{0}
$$

is the resistivity influenced by film thickness and surface-scattering coefficient.

$$
\rho_{c}=\frac{2}{k \ln 1 / k} \frac{(1-p) r}{s(1+p)(1-r)} \rho_{0}
$$

or

$$
\rho_{c}=\frac{\rho_{d}(\alpha \ll 1) \rho_{t}(k<1)}{\rho_{0}}
$$

If $\alpha \rightarrow 0$, then $\rho_{F} \rightarrow \rho_{t}$. For a single-crystal film external size effect alone is considered.

\subsection{Thick Film with Small Grains $(k>1, \alpha \gg 1)$}

Substituting Eq. (7) and (10) into Eq. (6) the 
expression for the film resistivity becomes

$$
\rho_{F}=\rho_{d}(\alpha \gg 1)+\rho_{c}(k>1, \alpha \gg 1)
$$

where

$$
\rho_{d}=\frac{4}{3} \frac{r}{s(1-r)} \rho_{0}
$$

is the film resistivity markedly affected by grainboundary scattering

$$
\rho_{c}=\frac{1}{2} \frac{1-p}{k} \frac{r}{s(1-r)} \rho_{0} \text { or } \rho_{c}=\frac{\rho_{d}(\alpha \gg 1) \rho_{t}(k>1)}{\rho_{0}}
$$

If $k \rightarrow \infty$, then $\rho_{F} \rightarrow \rho_{d}$. For very thick films characterized by a fine-grain structure and high values of $r$ internal size effect alone is taken into account.

\subsection{Thin Films of Fine-grained Structure $(k<1, \alpha \gg 1)$}

Introducing Eq. (8) and (10) into Eq. (6) gives

$$
\rho_{F}=\rho_{c}(k<1, \alpha \gg 1)
$$

where

$$
\rho_{c}=\frac{16}{9} \frac{1-p}{1+p} \frac{1}{k \ln 1 / k} \frac{r}{s(1-r)} \rho_{0}
$$

or

$$
\rho_{c}=\frac{\rho_{d}(\alpha \gg 1) \rho_{t}(k<1)}{\rho_{0}}
$$

In most cases films of that type, have the island structures and are, therefore, characterized by a conductivity different from that of the continuous films.

\section{COMPARISON WITH OTHER APPROXIMATE EXPRESSIONS}

Wedler and Wissmann ${ }^{5,6}$ have proposed the following expression for the electrical resistivity of a polycrystalline film

$$
\rho_{F}=\rho_{0}\left(1+K \frac{\lambda}{d}+\frac{3}{8} \frac{1-p}{k}\right)
$$

where $K$ is the phenomenological parameter describing the grain-boundary scattering effect. Thieme and Kirstein ${ }^{7}$ have demonstrated that if $k>1$ and $\alpha \ll 1$, then $K=3 / 21-r / r$. Combining this expression with Eq. (15) we have

$$
\rho_{F}=\rho_{0}\left(1+\frac{3}{2} \frac{\lambda}{d} \frac{r}{1-r}+\frac{3}{8} \frac{1-p}{k}\right)
$$

Eq. (16) equals Eq. (11) when $\rho_{c} \rightarrow 0$.

Mayadas and Shatzkes ${ }^{4}$ have considered the simultaneous action of surface scattering and grainboundary effects in terms of the function

$$
\Phi(k, p, \alpha)=G(\alpha)-A(k, p, \alpha)
$$

where

$$
\begin{gathered}
A=\frac{6}{\pi} \frac{1-p}{k} \int_{0}^{2 \pi} d \zeta \int_{1}^{\infty} \frac{\cos ^{2} \zeta}{H^{2}}\left(\frac{1}{t^{\prime 3}}-\frac{1}{t^{\prime 5}}\right) \\
\frac{1-\exp \left(-k t^{\prime} H\right)}{1-p \exp \left(-k t^{\prime} H\right)} d t^{\prime} \\
H=1+\alpha \cos ^{-1} \zeta\left(1-1 / t^{\prime 2}\right)^{-1 / 2}
\end{gathered}
$$

The dependence of film resistivity on size effects was also studied by Mola and Heras, ${ }^{8,9}$ starting from the relation

$$
\begin{aligned}
& \frac{\rho_{F}}{\rho_{0}}=\frac{1}{G(\alpha)-A(k, p, \alpha)} \text { or } \\
& \qquad \frac{\rho_{F}}{\rho_{g}}=\frac{G(\alpha)}{G(\alpha)-A(k, p, \alpha)}
\end{aligned}
$$

whereas

$$
\rho_{g}=\frac{\rho_{0}}{G(\alpha)}
$$

and $G(\alpha)-A(k, p, \alpha)=\Phi(k, p, \alpha)$ is the function derived by Mayadas and Shatzkes. ${ }^{4}$

Using the expressions of Eq. (19) for the electrical resistivity of a polycrystalline film, Tellier and Tosser ${ }^{11}$ have demonstrated that

$$
\frac{\rho_{F}}{\rho_{g}}=1+\frac{1-p}{k} h(\alpha)
$$

where $h(\alpha)$ is a function which takes into account the grain-boundary scattering. ${ }^{11}$ 
Based on Eq. (20), (21) and (11) we deduce that $h(\alpha)=3 / 8 G(\alpha)$. This is in agreement with the curves plotted by Tellier and Tosser. ${ }^{11}$ Other dependences between electron scattering and external size effect were studied by the same authors ${ }^{12}$ who have derived the same expression as the one referred to in this paper as (16).

\section{DISCUSSION AND CONCLUSIONS}

The films for which $t / \lambda \geqslant 1$, namely $200 \AA$ thick and thicker films are in general, cintinuous. The same holds for those films, for which the parameter $\propto$ is less than 1 . Usually $d / \lambda>1$, and the reflection coefficient $r \cong 0.2{ }^{4}$ From Table I follows that if $d / \lambda>1$, and $r \leqslant 0.2$, then $\alpha \ll 1,{ }^{14}$ where $\alpha$ is $d / \lambda$ and $r$ dependent, similarly as in Eq. (5). Hence for calculating the sizeeffect dependence of the film resistivity Eq. (11) may be employed. Generally, for $\alpha \rightarrow 0$ we may write

$$
F(k, p) G(\alpha) \rightarrow G(\alpha)-A(k, p, \alpha)
$$

because

$$
\begin{aligned}
& G(\alpha \rightarrow 0) \rightarrow 1 \\
& A(k, p, \alpha \rightarrow 0) \longrightarrow \frac{6}{\pi} \frac{1-p}{k} \frac{\pi}{4} \int_{1}^{\infty}\left(\frac{1}{t^{\prime 3}}-\frac{1}{t^{\prime 5}}\right) \\
& \frac{1-\exp \left(-k t^{\prime}\right)}{1-p \exp \left(-k t^{\prime}\right)} d t^{\prime} \\
& G(\alpha \rightarrow 0)-A(k, p, \alpha \rightarrow 0) \longrightarrow F(k, p)
\end{aligned}
$$

We can, therefore, expect that Eq. (6) and (19) will only slightly differ for $\alpha \ll 1$ and $k>1$.

Table II provides the values of $\rho_{F} / \rho_{0}$ calculated from Eq. (6) and from the results reported in Refs. 14,15 . The calculations were carried out for $0.024 \leqslant \alpha \leqslant 3$ and $0.01 \leqslant k \leqslant 50$ with $p=0$. In Table III, the $\rho_{F} / \rho_{0}$ calculation results are listed for $p=0.5$, while $\alpha$ and $k$ remained the same as in Table II. The deviation between these results and those obtained from Eq. (19) does not exceed $2 \%$ for $\alpha \ll 1$ and $k>1$ or $\alpha>1$ and $k \gg 1$, This deviation becomes considerable for $\alpha>1$ and $k \ll 1$, but is negligible for very thin and discontinuous films.

Eq. (6), which is a product of $F(k, p)$ and $G(\alpha)$, is more convenient for calculations than $\Phi(k, p, \alpha)$. As can be seen from Tables II and III, Eq. (6) is a good approximation of $k>1$ and $\alpha<1$, because $F(k, p) G(\alpha) \cong \Phi(k, p, \alpha)$.

The problem of electron scattering at external surfaces was also considered by A. A. Cottey. ${ }^{16}$ To describe this effect he has derived the function

$$
C(\mu)=\frac{3}{2} \mu\left\{\mu-\frac{1}{2}+\left(1-\mu^{2}\right) \ln \left(1+\frac{1}{\mu}\right)\right\}
$$

where

$$
\mu=\frac{k}{1-p}
$$

The expression of $C(\mu)$ for $\mu>1$ leads to

$$
C(\mu)=1-\frac{3}{8 \mu}+\frac{1}{5 \mu^{2}}-\ldots
$$

Neglecting high power terms gives $C(\mu) \cong F(k, p)$. Substituting $C(\mu)$ for $F(k, p)$ in Eq. (6) we obtain

$$
\frac{\rho_{F}}{\rho_{0}}=\frac{1}{C(\mu) G(\alpha)}
$$

Eq. (24) was employed for calculating the dependence of the strain coefficient of resistivity on size effects in copper films. ${ }^{17}$ The same equation was involved when studying the effect of the grain size on the resistivity of A1 films. ${ }^{18}$ Both studies have justified the agreement between theoretical and experimental results.

TABLE I

Values of function $G(\alpha)$

\begin{tabular}{lllllllll}
\hline$r$ & $\stackrel{d}{\lambda}$ & 10 & 5 & 2 & 1 & 0,5 & 0,4 & 0,33 \\
\hline 0,2 & $\alpha$ & 0,024 & 0,050 & 0,124 & 0,250 & 0,5 & 0,624 & 0,750 \\
& $G / \alpha /$ & 0,965 & 0,931 & 0,847 & 0,737 & 0,588 & 0,534 & 0,490 \\
0,5 & $\alpha$ & 0,1 & 0,2 & 0,5 & 1 & 2 & 2,5 & 3 \\
& $G / \alpha /$ & 0,872 & 0,776 & 0,588 & 0,420 & 0,268 & 0,227 & 0,197 \\
\hline
\end{tabular}




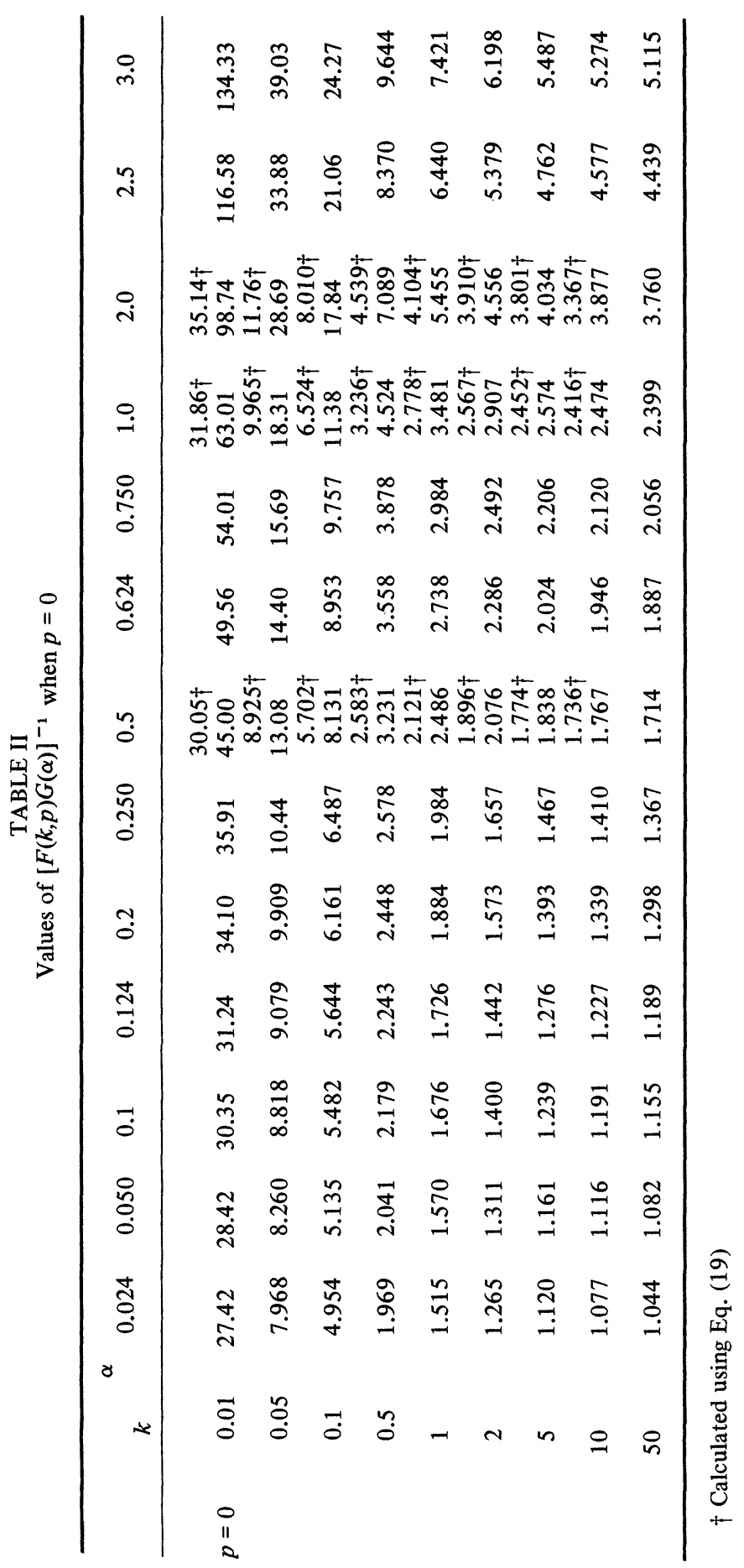




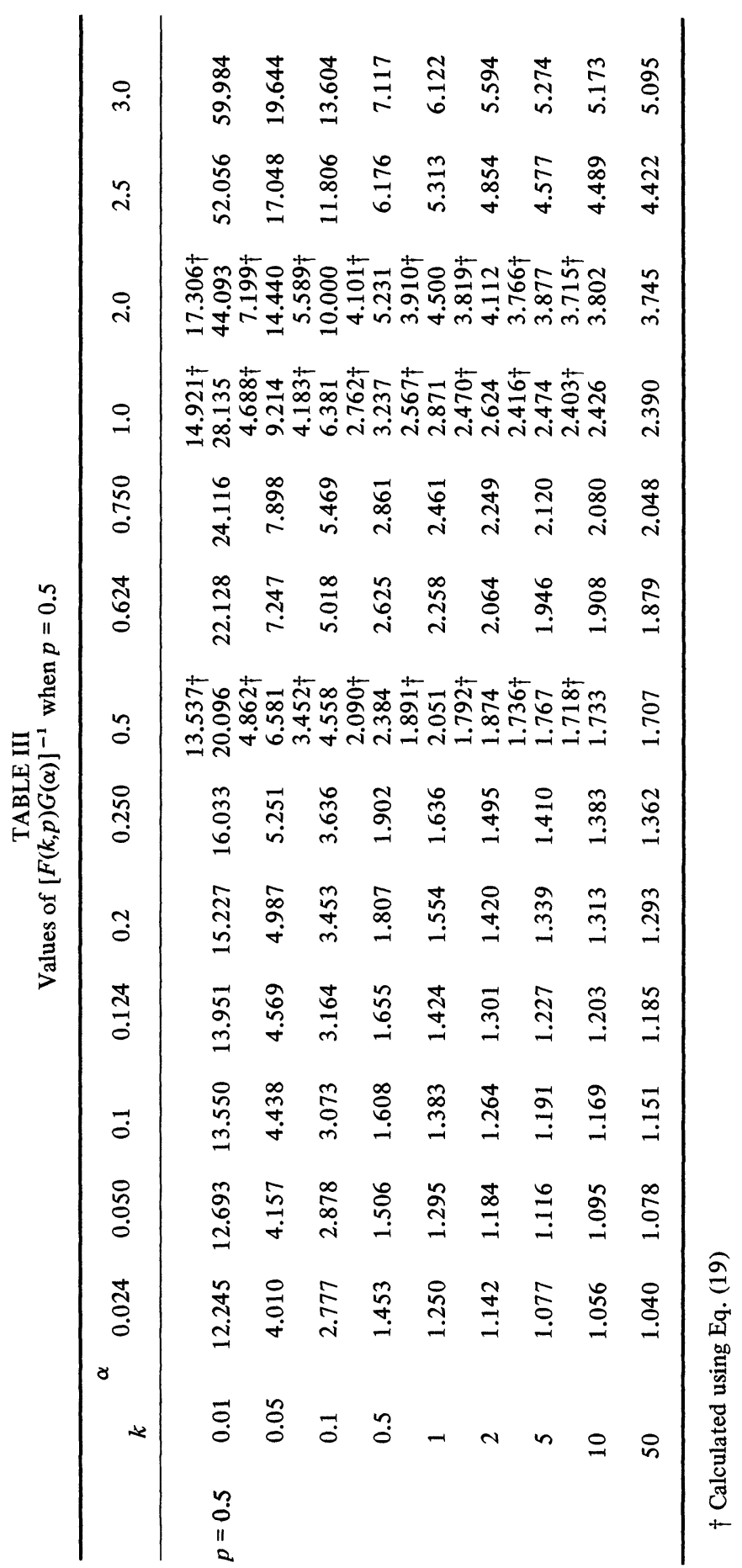




\section{ACKNOWLEDGEMENT}

The author is indebted to Professor C. Wesolowska for her interest and encouragement, and to Doctor E. DobierzewskaMozrzymas for many useful discussions.

\section{REFERENCES}

1. K. Fuchs, Proc. Cambridge Phil. Soc., 34, 100 (1938).

2. E. H. Sondheimer, $A d v$. Phys., 1, 1 (1952).

3. A. F. Mayadas, M. Shatzkes and J. F. Janak, Appl. Phys. Lett., 14, 345 (1969).

4. A. F. Mayadas and M. Shatzkes, Phys. Rev., B, 1, $1382(1970)$.

5. G. Wedler and P. Wissmann, Ber. Bunsenges, Phys. Chem., 74, 934 (1970).

6. P. Wissmann, Thin Solid Films, 5, 329 (1970).

7. F. Thieme and W. Kirstein, Thin Solid Films, 30, 371 (1975).
8. E. E. Mola, J. Borraja and J. M. Heras, Surface Science, 34, 561 (1973).

9. E. E. Mola and J. M. Heras, Thin Solid Films, 18, 137 (1973).

10. E. E. Mola and J. M. Heras, Electrocomponent Science and Technology, 1, 77 (1974).

11. C. R. Tellier and A. J. Tosser, Thin Solid Films, 33, L19, (1976).

12. C. R. Tellier and A. J. Tosser, Electrocomponent Science and Technology, 3, 165 (1976).

13. L. A. Moraga and A. Vilche, Thin Solid Films, 38, 117 (1976).

14. B. S. Verma, G. L. Malhotra and S. K. Sharma, Thin Solid Films, 6, R9 (1970).

15. S. Soffer, J. Appl. Phys., 36, 3947 (1965).

16. A. A. Cottey, Thin Solid Films, 1, 297 (1968).

17. F. Warkusz, Thin Solid Films, 41, 261 (1977).

18. E. Dobierzewska-Mozrzymas and F. Warkusz, Thin Solid Films, 43, 267 (1977). 

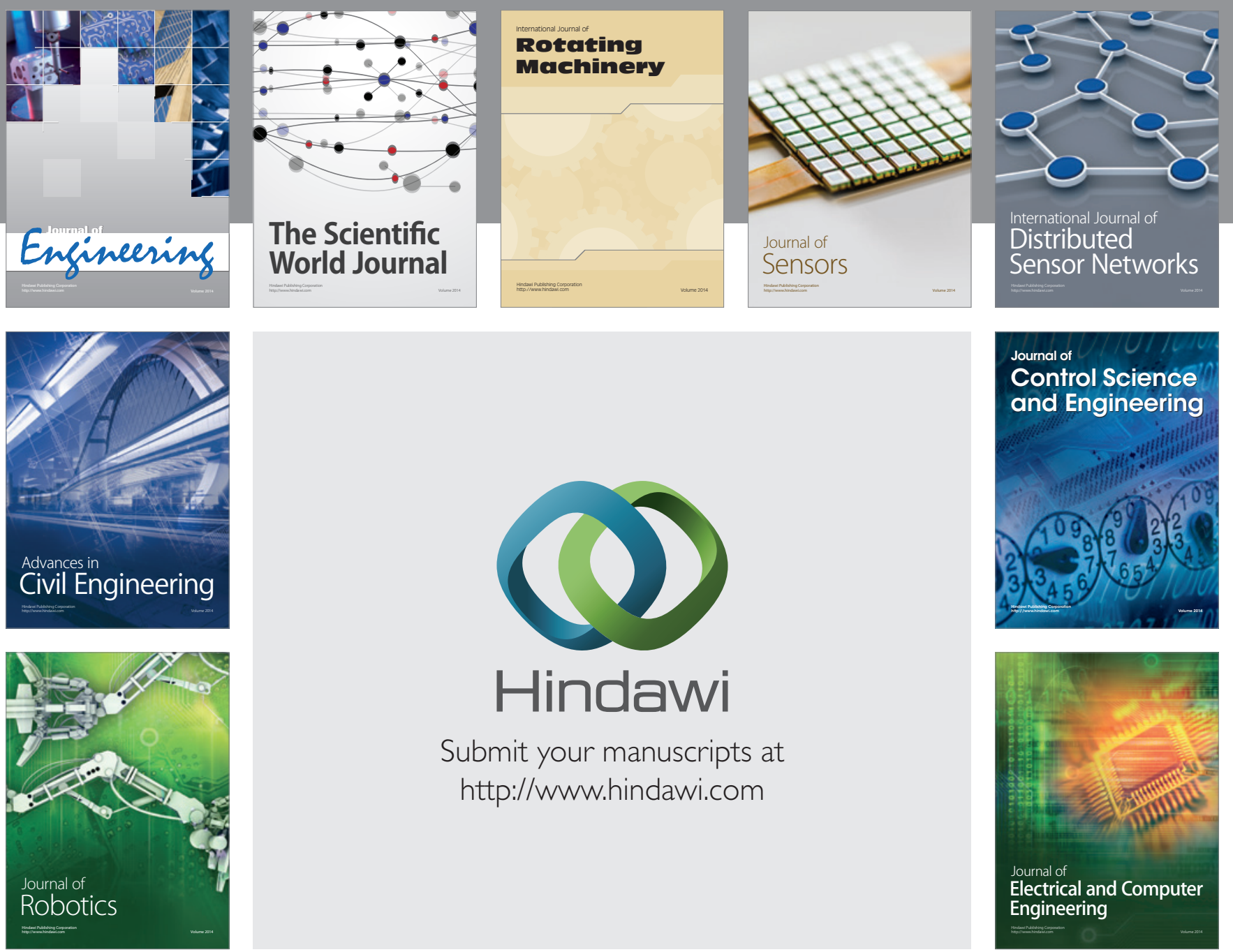

Submit your manuscripts at

http://www.hindawi.com
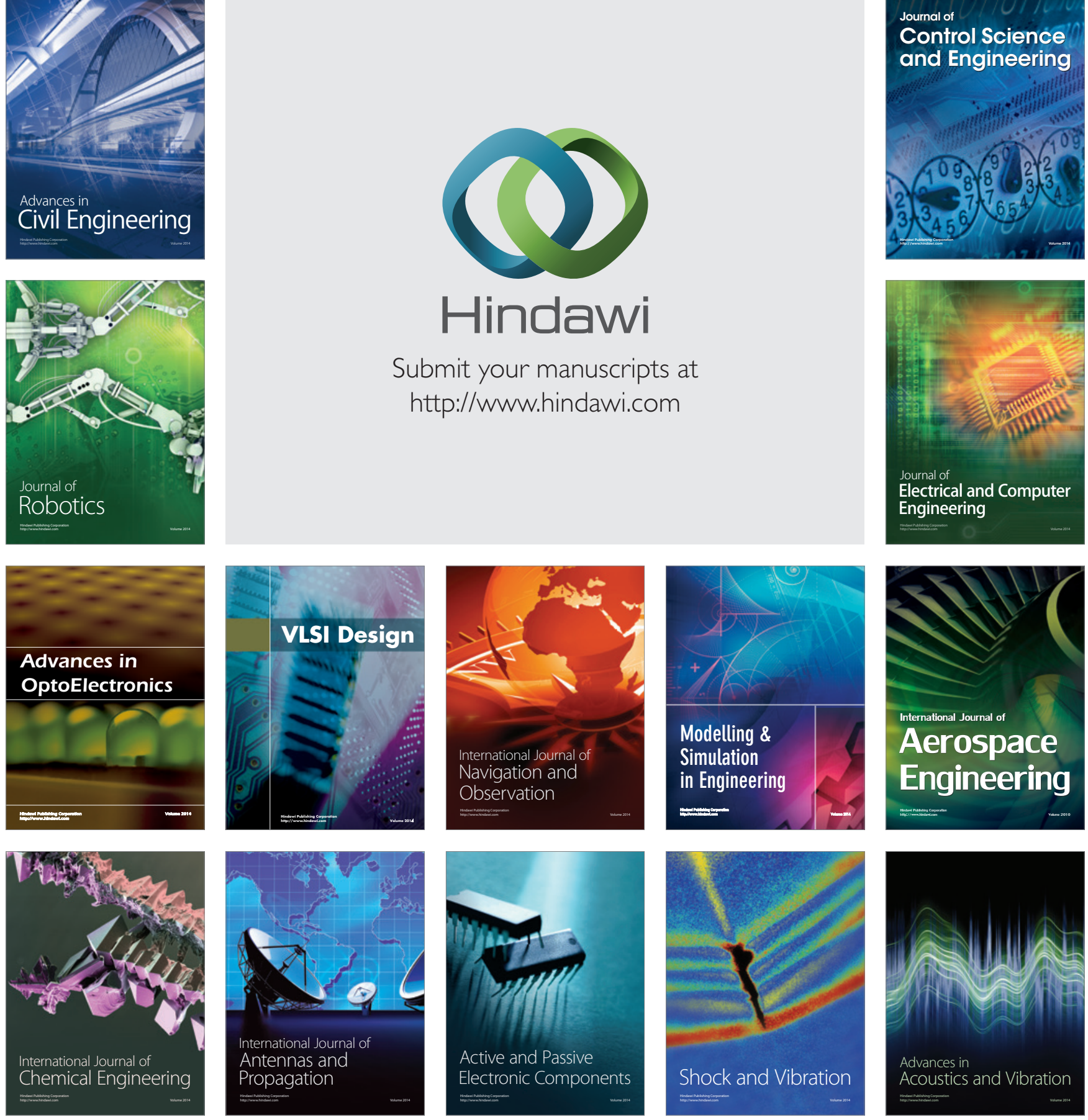